\title{
Consequences of Adolescent Ethanol Consumption on Risk Preference and Orbitofrontal Cortex Encoding of Reward
}

\author{
Matthew Stephen McMurray', Leslie Renee Amodeo' and Jamie Donahey Roitman ${ }^{*, 1,2}$ \\ 'Department of Psychology, University of Illinois at Chicago, Chicago, IL, USA; '2Laboratory of Integrative Neuroscience, University of Illinois at \\ Chicago, Chicago, IL, USA
}

\begin{abstract}
Critical development of the prefrontal cortex occurs during adolescence, a period of increased independence marked by decision making that often includes engagement in risky behaviors, such as substance use. Consumption of alcohol during adolescence has been associated with increased impulsivity that persists across the lifespan, an effect which may be caused by long-term disruptions in cortical processing of rewards. To determine if alcohol consumption alters cortical encoding of rewards of different sizes and probabilities, we gave rats limited access to alcohol in gelatin during adolescence only. In adulthood, we recorded the electrophysiological activity of individual neurons of the orbitofrontal cortex while rats performed a risk task that varied the level of risk from day-to-day. Rats that had consumed higher levels of alcohol showed increased risk preference in the task compared with control and low alcohol-consuming rats. Patterns of neuronal responses were identified using principal component analysis. Of the multiple patterns observed, only one was modulated by adolescent alcohol consumption and showed strongest modulation after reward receipt. This subpopulation of neurons showed blunted firing rates following rewards in alcohol-consuming rats, suggesting a mechanism through which adolescent alcohol exposure may have lasting effects on reward processing in the context of decision making. The differences in OFC responses between high alcohol consumers and control animals not given access to alcohol support the idea that, regardless of potential variability in innate alcohol preferences, voluntary consumption of alcohol during adolescence biases choice patterns longitudinally through alterations in cortical function.

Neuropsychopharmacology (2016) 4I, 1366-1375; doi:I0.1038/npp.2015.288; published online 7 October 2015
\end{abstract}

\section{INTRODUCTION}

Consumption of alcohol during adolescence may seriously impact the developing cortico-striatal circuitry involved in decision making and reward processing (Bava and Tapert, 2010; Crews et al, 2007; Doremus-Fitzwater et al, 2010). Adolescent alcohol exposure has been associated with deficits in neurogenesis (Crews et al, 2006) and an increase in neurodegeneration (Nixon et al, 2010) in a number of brain regions, and may have long-term effects on behavior through these means. Synaptic pruning, one of the most prolific developmental processes occurring during this period, is likewise altered by adolescent alcohol consumption (Selemon, 2013), particularly in the glutamatergic cell populations that project from cortical to striatal regions. Thus, alcohol consumption during adolescence has been associated with disruptions to many normal developmental processes, potentially disturbing the maturation of higher-order executive function that relies on this circuitry.

* Correspondence: Dr JD Roitman, Department of Psychology, University of Illinois at Chicago, 1007 W Harrison St MC 285, Chicago, IL 60607, USA, Tel: + | 312355 |458, Fax: + | 312 4I3 4I22,

E-mail: jroitman@uic.edu

Received 4 May 20I5; revised 17 July 2015; accepted 8 August 2015; accepted article preview online 15 September 2015
The orbitofrontal portion of the prefrontal cortex (OFC) in particular has been found to have a role in decisions based on outcome value and reward expectations (Kepecs et al, 2008; McDannald et al, 2014; Padoa-Schioppa and Cai, 2011; Stalnaker et al, 2014). When rewards are uncertain, the magnitude of response in OFC has been shown to modulate proportionally with risk (Tobler et al, 2009), and lesions of OFC disrupt decisions that involve risk (Hsu et al, 2005). We previously found the level of OFC activity following delivery of 'certain' and 'risky' rewards varied with reward size, probability, and preference for a risky reward option in rats (Roitman and Roitman, 2010).

To examine how perturbations of prefrontal cortex development by consumption of alcohol during adolescence might alter decisions and their neural correlates, we measured adult animals' risk preference and OFC responses to probabilistic rewards following voluntary consumption of alcohol during adolescence. Adolescent rats were given daily access to ethanol in gelatin form for a limited period of time at the onset of the dark cycle to encourage a binge-like pattern of consumption. This pattern of consumption has previously been shown to result in a bias towards risky outcomes in animals tested during adolescence (McMurray et al, 2014). Here, following the adolescent period, alcohol was no longer given to rats, and we examined preferences for risky payoffs across a range of probabilities, while simultaneously recording OFC activity using in vivo 
electrophysiology. Previous studies have shown enhanced risk-preference associated with altered neural signaling following similar adolescent alcohol consumption (Clark et al, 2012; Nasrallah et al, 2009, 2011). We therefore hypothesized that greater modulation of OFC activity in response to rewards under conditions of risk would be associated with enhanced preference for the risky choice option across levels of risk.

\section{MATERIALS AND METHODS}

\section{Subjects}

Eighteen male Sprague Dawley rats (Charles River Laboratory, Chicago, IL) arrived in the laboratory on postnatal day 22 (PD 22). Rats were housed in groups of four (two control and two ethanol, detailed below) in polycarbonate cages $(56 \times 34 \times 22 \mathrm{~cm})$ and provided lab chow (LabDiet 5012, Richmond, IN) and water ad libitum until adulthood (PD 90). As adults, animals were singly housed and food-restricted no $<90 \%$ of their free-feeding weight. The colony was maintained on a 12:12 light/dark cycle (0700-1900 hours) with behavioral testing conducted in a separate experimental room during the light phase of the cycle. Animals were treated in accordance with the guidelines put forth by the National Institutes of Health and under the approval of the Animal Care Committee of the University of Illinois at Chicago.

\section{Adolescent Ethanol Access}

As previously described (McMurray et al, 2014), EtOH was available during access hours in a gel comprised of distilled water, 2.5\% Knox gelatin (Kraft Foods, Northfield, IL), $10 \%$ Polycose (Abbot Laboratories, Columbus, Ohio), and $10 \%$ EtOH by weight. A non-ethanol gelatin was presented to control animals $(n=6)$ throughout exposure. To account for the calorie discrepancy between Ethanol and Control gelatin, the percentage of polycose was increased in the Control diet, such that the diets were calorie-matched by volume. Gelatin was provided to all animals using a modified 'drinking-inthe-dark-multiple-scheduled-access' paradigm (Bell et al, 2006, 2011; Peris et al, 2006), which permitted access at the onset of the dark cycle for $12 \mathrm{~h}$ on PD 29 and PD 30, $6 \mathrm{~h}$ on PD 31, 3 h on PD 32, and $1 \mathrm{~h}$ daily from PD 33 to PD 49. On the pre-exposure day (PD 29), all animals were given $12 \mathrm{~h}$ overnight access to control gelatin to reduce neophobia during the first EtOH gelatin access period. Of the 18 animals tested, all animals consumed at least $5 \mathrm{~g}$ of gelatin during this period. During the daily access periods, mesh dividers were inserted into the home cage to allow animals social contact, while maintaining separate access to gelatin for accurate measurement of consumption. After each daily access period, jar weights were recorded and gelatin consumption was calculated in $\mathrm{g} / \mathrm{kg}$ for each animal, using the individual body weights measured that day. On the last night of gelatin access (PD 49), blood was taken via tail nick to measure BELs as previously described (McMurray et al, 2014). Following access to EtOH or Control gelatin throughout adolescence, animals began behavioral testing as adults 40 days following their last day of ethanol access (PD 90).

\section{Risk Preference Assessment}

Behavioral testing was conducted in operant chambers (Med Associates, St Albans, VT) equipped with a central pellet dispenser and two levers located on either side of the central port, with cue lights located above each lever. A house light was positioned at the rear of the chamber and an infrared beam marked entries into the pellet dispenser. One of the two levers was associated with a small, but certain, payoff (two $45 \mathrm{mg}$ sucrose pellets; BioServ, Frenchtown, NJ) and the other was associated with a potentially large, but risky, outcome (four (Win) or zero (Lose) $45 \mathrm{mg}$ sucrose pellets). The spatial associations of each lever were held constant for each rat across all of its sessions (ie, the left lever was always certain for a single animal), but were counterbalanced evenly across animals within each group. In addition, all animals were first trained on a version of the task with $100 \%$ probability of risky reward until at least $80 \%$ of the lever presses occurred for the larger reward. Combined with random lever side assignment, this ensured that any innate lever preference was overruled by a preference for the side associated with larger reward.

In each session of the risk task, rats first completed a block of Forced Response trials in which the certain and risky levers were presented in alternation. Forced Response trials were presented to ensure experience with the ratio of reward deliveries and omissions for risky responses (ie, three reward omissions for every trial rewarded in a $25 \%$ session, presented pseudo-randomly). A Free Choice block then followed, in which both options were presented simultaneously on each trial, and rats choose freely between them for a maximum of 150 choice trials. One-hundred percent of 'certain' responses resulted in small reward delivery, but the probability of risky reward delivery varied randomly from session-to-session $(12.5,25$, or $50 \%)$ and was held constant within each daily session. The expected values for certain and risky choices were equal for $50 \%$ sessions (2 vs 2), but favored certain choices for 25\% (2vs 1) and 12.5\% (2 vs 0.5 ) sessions, thus providing conditions in which biases towards potential large wins (or insensitivity to loss) would be more detectable.

Each trial within a session was initiated when the rat poked its nose into the central port, leading to the illumination of the cue light(s) for $2 \mathrm{~s}$ to indicate the option(s) available, followed by the extension of the lever(s) into the chamber (Supplementary Figure S1). Once a lever was pressed, the cue light(s) extinguished, the lever(s) retracted, and the reward (or omission) immediately followed. Ten seconds after the reward was delivered, the house light was extinguished for a varibale amount of time (1-5s), after which the light would be reilluminated to indicate the start of the next trial. Reward ommission was denoted by the lack of a reward, but no other discerning cues.

All animals were tested once per day, on a single risky reward probability per day. Risk preference was calculated as a percentage of presses on the risky lever for each session. Overall behavioral performance was then assessed via two-way (treatment group $\times$ risky probability) ANOVA, followed by Tukey posthoc tests where appropriate. 


\section{Surgical Procedures}

Custom-designed stainless steel Teflon insulated electrode arrays (MicroProbes, Gaithersburg, MD), organized into two columns of four microwires ( $50 \mu \mathrm{m}$ diameter; tip separation $0.25 \mathrm{~mm}$ ), were stereotaxically guided bilaterally into the OFC ( $\mathrm{AP}+3.2, \mathrm{ML} \pm 3.0$ relative to bregma, and $-4.0 \mathrm{~mm}$ relative to skull surface) under ketamine/xylazine anesthesia. Ground wires for each array were inserted into the brain at a distal location. Connectors were anchored to the skull via stainless steel screws and dental acrylic. Arrays were implanted 1 week before recording, allowing animals sufficient time to recover, ensuring normal behavioral performance.

\section{Electrophysiological Recording}

Electrophysiological recordings of OFC activity were completed in adult animals. Each animal was connected to a flexible recording cable attached to a motorized commutator (Plexon, Dallas, TX), to allow for relatively free movement. Electrical signals in the vicinity of the electrode tips were amplified and transduced via the OmniPlex system (Plexon, Dallas, TX). In addition, the time of trial events, such as cue and lever presentation, lever press, and sucrose pellet delivery, were time-stamped onto the neural spike data. During recording, individual waveform statistics, including principal components and inter-spike intervals, were used to identify waveforms belonging to individual neurons (PlexControl), which were then subsequently refined offline (Offline Sorter). The data are exported to Matlab (Mathworks) and SAS v9.2 for analysis as detailed below. At the conclusion of testing, electrode placements are verified histologically (Figure 1). Based on these data, 24 units were excluded due to electrode location. These misplacements occurred in two animals (one Control and one EtOH-low) and all were located in the Olfactory Bulb. In addition, 1 unit was excluded due to an insufficient number of trials and 52 units were excluded due to mean firing rates that indicated the possibility of poor separation of multipleunit activity or artifact from other sources of noise $(>20 \mathrm{~Hz}$ sustained across the entire session). This left a total of 461 units across the 18 animals.

\section{Electrophysiological Analyses}

Only data from sessions in which the probability of risk payoff was 25 and $50 \%$ were included in the electrophysiological analyses. Sessions with $12.5 \%$ payoff probability resulted in too few Win outcomes to be compared statistically, as animals overwhelmingly favored the Certain option on these sessions. Except where indicated otherwise, all analyses of electrophysiological data were conducted on normalized data. For each unit, the mean firing rate and SD of firing rate were calculated across the entire recording session. For each trial, raw firing rate was calculated in $0.5 \mathrm{~s}$ bins, from -10 to $+15 \mathrm{~s}$ aligned to the time of lever press. Firing rate for each trial, in each time bin, was $\mathrm{z}$ normalized using that unit's mean firing rate and SD for the entire session.

Because there were several temporally distinct trial events with the potential to cause modulations in neural activity

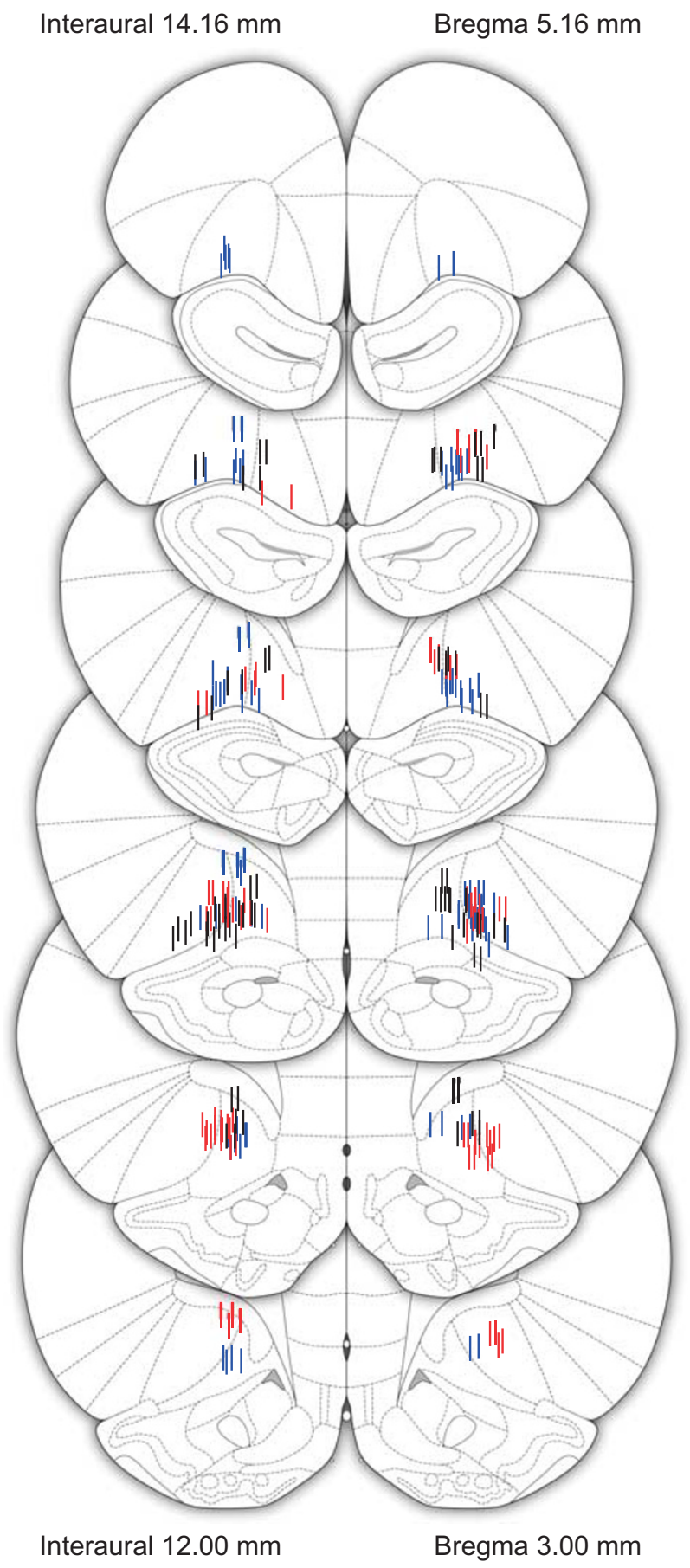

Figure I Location of electrophysiological recording sites. Owing to vertical wire movement during the extraction process, vertical lines are used to indicate the approximate location of each electrode tip. Control=black; $\mathrm{EtOH}-\mathrm{low}=$ blue; $\mathrm{EtOH}$-high $=$ red.

(nose poke to initiate trial, illumination of cue lights, lever extension, lever press, reward), principal component analysis was used to identify patterns of neural activity across the population that accounted for variability in responses over the course of the entire trial. For each neuron, the average normalized firing rate from $10 \mathrm{~s}$ before lever press to $15 \mathrm{~s}$ after was calculated for all rewarded trials (both Certain and 
Win). Principal component analysis was performed on the normalized firing rates, and identified five primary patterns of activity (eigenvalues $>1$ ) across the entire neuronal population, which collectively explained $>70 \%$ of the variance in firing patterns. Using least squares fit, we then assigned each unit to one of the five patterns of activity ('Types') by comparing the fit of each of the five patterns (scaled to that unit's minimum and maximum firing rates) to the unit's average firing rate across rewarded trials. This method accounted for between-group differences in mean firing rates, while still allowing the pattern of activity to drive functional grouping. The number of units assigned to each type were compared across groups using $\chi^{2}$, followed by $z$ tests for proportions, with Bonferroni-corrected $p$-values. Once assigned, unit Type was used to identify neurons for comparison of neural responses for different consumption groups (Control, EtOH-low, EtOH-high), session probabilities $(25 \%, 50 \%)$, and outcomes (Certain, Win, Lose), which were compared using repeated measures generalized linear mixed models, and corrected for multiple comparisons using the Tukey-Kramer method. Raw baseline firing rate ( -10 to -5 s pre-lever press) of each unit type was compared using two-way analysis of variance (group $\times$ type). We used regression test to model the effect of outcome (win/loss and reward magnitude), anatomical location of electrode (ipsilateral/contralateral to risky lever, or anterior/posterior site) and group on neural response, and the effect of neural response and group on risk preference. We also tested the null hypothesis that consumption level (group) did not affect OFC firing rate or risk preference using an F-test for nested models.

\section{RESULTS}

\section{Ethanol Consumption and Adolescent Development}

Of the twelve animals given access to EtOH gelatin, half of these voluntarily consumed a daily average of $1.88 \pm 0.11 \mathrm{~g} / \mathrm{kg}$ of EtOH during the hour long access period, and were thus grouped together as EtOH-high $(n=6)$. The remaining animals consumed a daily average of $0.65 \pm 0.10 \mathrm{~g} / \mathrm{kg}$ of EtOH were grouped together as EtOHlow $(n=6)$. The intake of the two groups was nonoverlapping, with a mean split at $1.25 \mathrm{~g} / \mathrm{kg} \mathrm{EtOH}$ daily (Supplementary Figure S2A). Concurrent with animals' growth from PD 33-49, their gelatin consumption increased slightly within each of the three groups. At the completion of the final day's access period (PD 49), EtOH-high animals demonstrated significantly higher blood ethanol levels (BELs) than EtOH-low $(t=3.99, p<0.01$, Supplementary Figure S2A). On average, EtOH-high animals attained BELs approximately at the criterion for binge drinking (80 $\mathrm{mg} \%$, National Institute on Alcohol Abuse and Alcoholism, 2004), while BELs from EtOH-low animals were significantly lower. Across the 1-hour access days (PD 33-49), Control animals ate considerably more gelatin than either of the $\mathrm{EtOH}$ groups $(\mathrm{F}(2,347)=23.6, \quad p<0.001)$, while EtOH intake differed between EtOH-high and EtOH-low $(\mathrm{F}(2,195)=66.9$, $p<0.001)$. The differential patterns of gelatin intake did not result in significant differences in body weight across adolescence. After the final access period on PD 49, gelatin access was terminated for the remainder of the study. All adult behavioral and electrophysiological testing, which began on PD 90, therefore occurred during a forced abstention state.

\section{Adolescent Ethanol Consumption Alters Risk Preference}

Regardless of treatment group, all animals selected the risky lever less frequently as probability payoff decreased (Figure $2 \mathrm{a} ; \mathrm{F}(2,49)=17.6, p<0.001$ ), suggesting that they understood the changing risk contingencies from session-tosession. EtOH-high animals showed elevated preference for the risky option compared with both Controls and EtOH-low animals (Figure $2 \mathrm{~b}$ and $\mathrm{c}$ ), regardless of probability of risky payoff $(\mathrm{F}(2,49)=11.2, p<0.001)$. This pattern of behavior resulted in a lower effective 'rate of return' for each trial, which EtOH-high offset by completing a higher number of trials per session $(\mathrm{F}(2,49)=5.1, p=0.02$, Supplementary Figure S3A), resulting in a similar number of pellets earned per session (ns). Controls and EtOH-low animals did not differ in risk preference, nor the number of trials completed. In addition, within behavioral sessions, the proportion of risky presses during the Free Choice block remained stable from beginning to end, regardless of probability (Supplementary Figure S3B-D). Thus, following experience with the reward contingencies during the Forced block, animals maintained a consistent risk preference in any given session, despite potential changes in satiation as they completed trials and consumed more sucrose pellets.

\section{Patterns of Adult OFC Activity Are Altered in Rats that Consumed EtOH as Adolescents}

During behavioral sessions, we recorded the activity of 461 individual neurons in OFC using chronically implanted multi-wire electrodes (159 from Controls, 167 from EtOHlow, and 135 from EtOH-high; Figure 1). Across the population of neurons, we identified the five principle components that accounted for the greatest variability in patterns of OFC activity in response to task events (Supplementary Figure S4A and B). For each of these principle components (Types), the responses on rewarded (Supplementary Figure S5, left) and non-rewarded (Supplementary Figure S5, right) trials did not differ between 50 and $25 \%$ risky sessions, so neural responses were combined across these sessions for all analyses.

Figure 3a (left) shows the average activity of each neuron identified by type, based on its response on rewarded trials. Activity for each neuron on non-rewarded trials is plotted separately (Figure 3a, right). Types 1, 2, and 5 showed modulation before the lever press, with Type 2 neurons showing increased activity, while Types 1 and 5 decreased. After the lever press, when each trial's outcome was disclosed (outcome is delivered immediately on lever press), the direction of the neural response differed as well (Figure $3 \mathrm{~b}$ ), with Type 1 increasing following reward delivery, and Types 4 and 5 decreasing. Types that differentiated rewarded from non-rewarded trials $(1,4$, and 5) showed weaker modulations on non-rewarded trials compared with rewarded. Overall, Types of OFC neurons showed complex patterns of activity during task performance that were comprised of both increases and decreases during the pre-press and the 

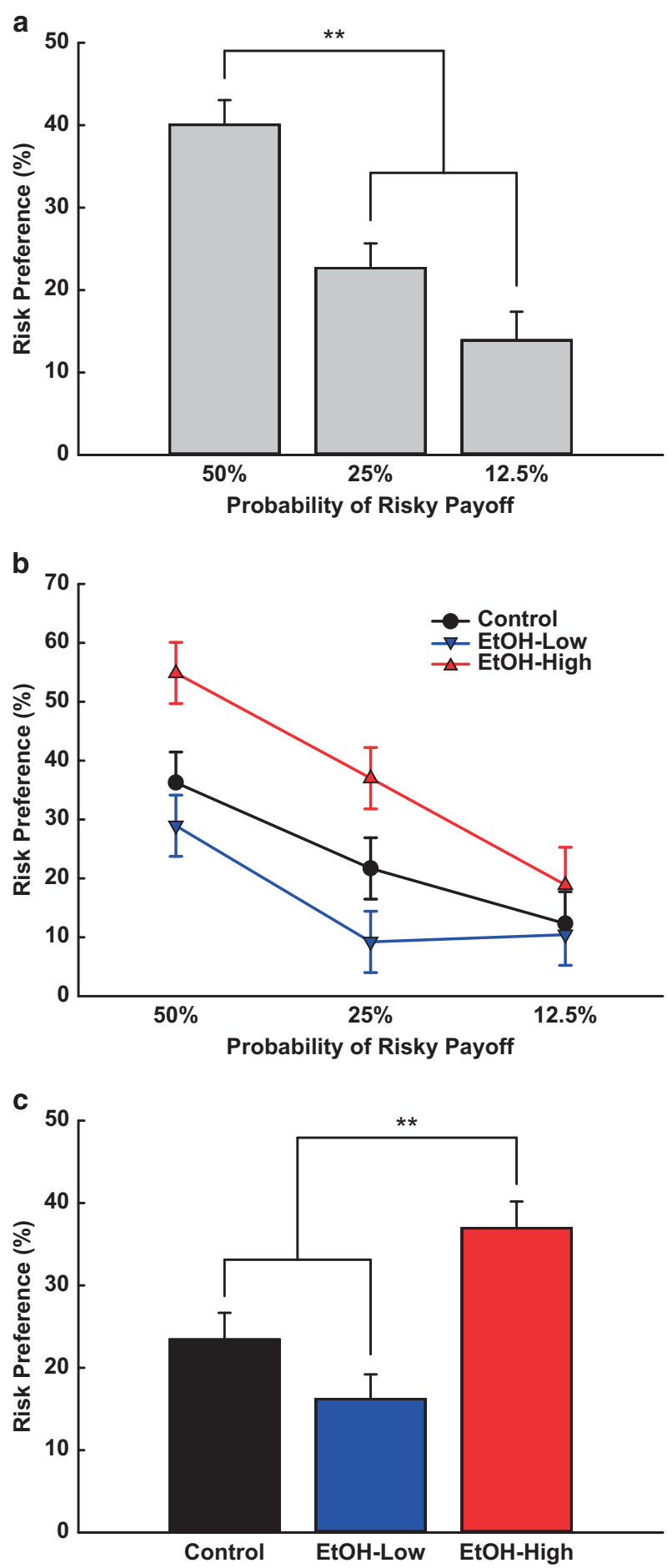

Figure 2 Effects of adolescent ethanol intake on risk preference. (a) All animals reduced their preference for the risky lever on free-choice trials as the probability of risky payoff decreased $(F(2,49)=17.6, p<0.00 I)$. (b) Risk preference across groups as a function of the probability of risky payoff. (c) $\mathrm{EtOH}$-high demonstrated elevated risk preference across probability levels $(\mathrm{rmANOVA}, F(2,49)=|| .2, p<0.001)$. ** $p<0.00 \mid$.

post-reward periods and response differed between rewarded and non-rewarded trials.

The proportions of neurons assigned to each neural response Type differed between ethanol-treatment groups $\left(\mathrm{X}^{2}(8, N=461)=29.1, p \leqslant 0.01\right.$, see Table 1$)$. Control animals had proportionately more Type 1 units than EtOH-high $(z=3.34, p \leqslant 0.01)$ and EtOH-low $(z=3.85, p<0.01)$. In addition, EtOH-high had proportionately more Type 2 units than both Controls $(z=5.98, p<0.01)$ and EtOH-low $(z=9.47, \quad p<0.01)$, while Controls had more than EtOH-low $(z=2.96, p \leqslant 0.05)$. Lastly, EtOH-high animals had higher proportions of Type 5 units than EtOH-low $(z=5.03, p<0.01)$. Despite the differing proportions of units assigned to each Type, there was no difference in baseline activity levels ( -10 to $-5 \mathrm{~s}$ before the lever press) between or within Unit Types, or between the treatment groups (Supplementary Figure S6).

Type 1 units, which were modulated during the period following reward delivery, were the only Type with activity patterns that depended on both $\mathrm{EtOH}$ consumption group and outcome (Supplementary Figure S7 for Types 2-5). For each outcome, the magnitude of Type 1 neuronal response differed between consumption groups. On Certain trials, OFC neurons in Control and EtOH-low animals showed a robust elevation of neural firing rate following reward delivery, while elevations in EtOH-high animals were significantly reduced compared with Controls (Figure 4a, indicated by horizontal bar along bottom axis). A similar pattern was observed on Win trials, with OFC neurons in Control and EtOH-low animals showing the strongest increases in response during the reward-processing interval and a reduction in response in EtOH-high subjects (Figure 4b). On Lose trials, the post-lever-press increase in activity was absent for all groups, and no differences between groups were evident (Figure 4c).

Within each treatment group, the pattern of outcome encoding by Type 1 neurons differed. In Control animals, activity following Certain presses increased earlier, but responses to both Certain outcomes and Wins were elevated compared with Losses over a prolonged period (Figure 4d). Similarly, in EtOH-low animals, OFC responses did not discriminate between Certain rewards and Wins, but both were elevated over Losses (Figure 4e). This elevation persisted for a shorter duration than in Controls. However, in the EtOH-high group, both the degree of modulation and the pattern of neural responses differed substantially from those observed in the other groups (Figure 4f). In these animals, Wins differed from both Certain and Lose outcomes; however, these differences occurred shortly after reward delivery and Wins were encoded with a reduction in firing rate from baseline during the significant periods, while all other groups encoded Wins with increases in firing rates.

The time course of Type 1 activity (Figure 4) shows that both consumption group and trial outcome caused modulations in activity over an extended period following reward delivery. The average modulations during the period +5 to $+8 \mathrm{~s}$ after reward are shown inset in each panel. During this epoch, OFC firing rate was modulated by whether or not the trial was rewarded (increase of 0.40 for reward, CI: $0.26-0.54$, $(\mathrm{F}(1,429)=40.06, p<0.0001))$, but this relationship was not improved by taking into account reward magnitude (change of -0.02 per pellet, $\mathrm{CI}$ : -0.07 to 0.01 , $(\mathrm{F}(1,428)=1.68, p=0.20))$. Responses also depended on the side of recording site (increase of 0.12 for sites ipsilateral to risky lever, CI: 0.03-0.18; $(\mathrm{F}(1,428)=28.49, p<0.0001))$. However, firing rate was not significantly modulated by anterior to posterior location of recording wire (change 
a

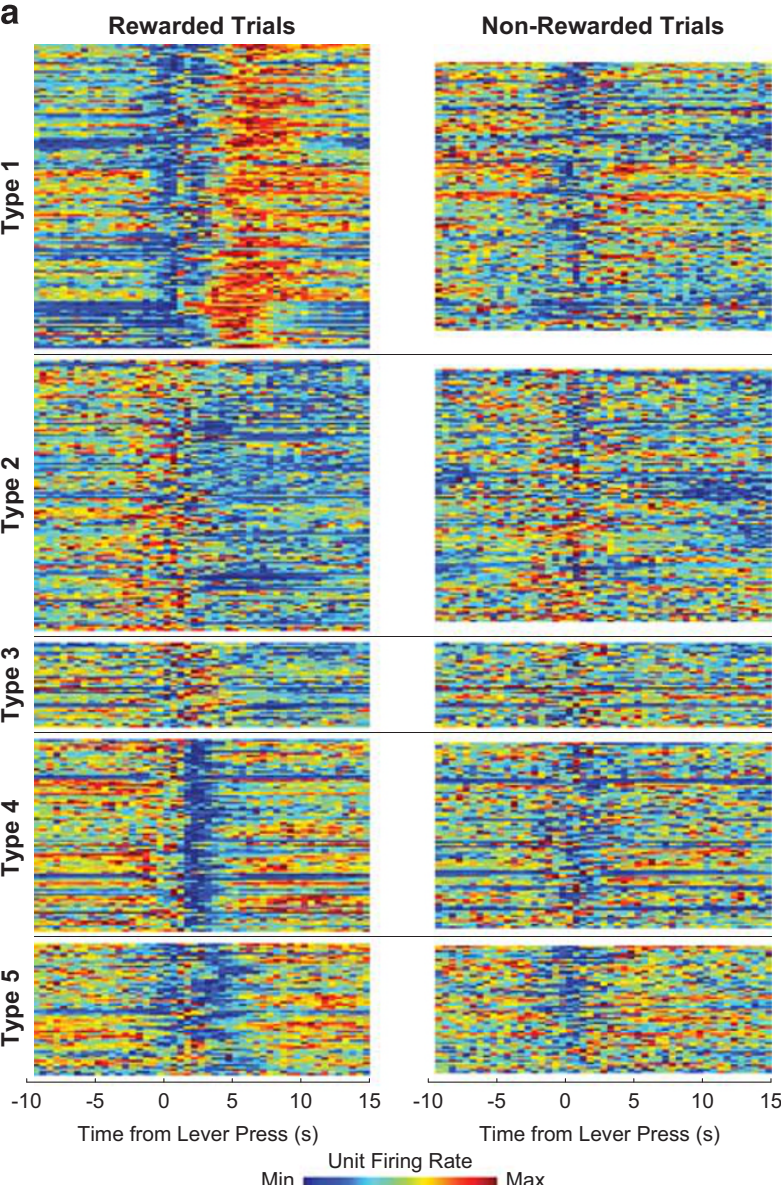

b
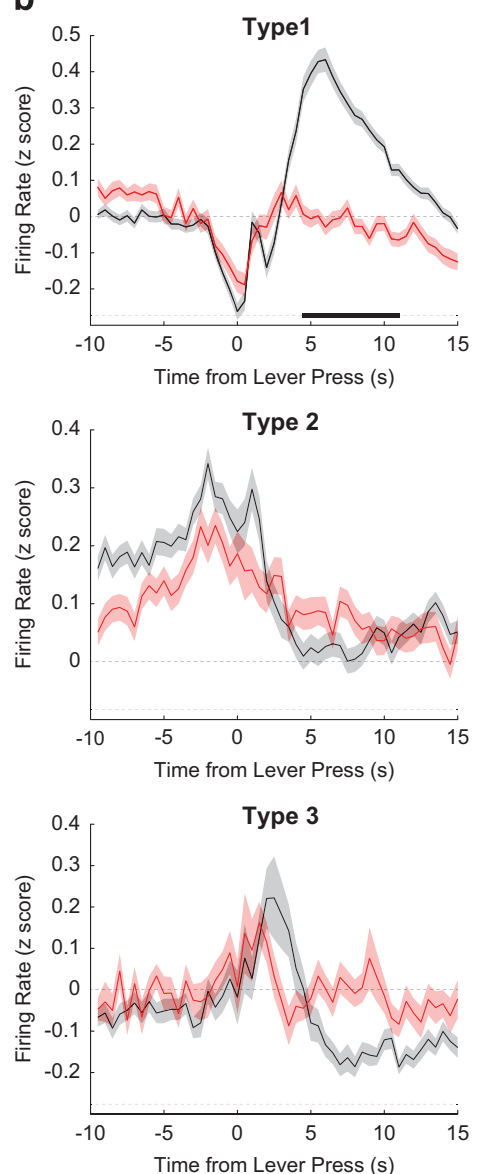
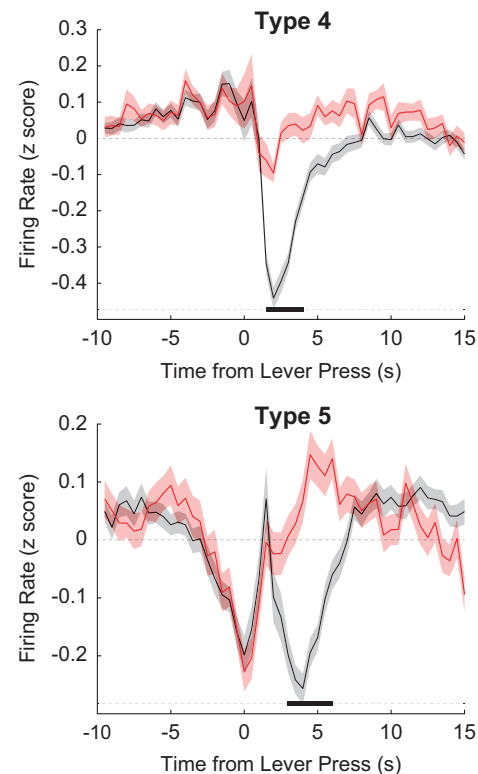

Figure 3 Patterns of electrophysiological activity in the OFC by functional Unit Type. Types were identified using Principle Component Analysis of activity patterns during rewarded free-choice trials. All data are shown aligned to the time of lever press/choice (time $=0$ ). Reward delivery $($ or omission) immediately followed the lever press. (a) Heat maps of activity patterns, in which each row represents the average activity pattern of a single neuron across trials, with cooler colors indicating levels of activity close to the minimum for that unit, while warmer colors indicate activity levels close to the maximum for that unit (based on normalized firing rates). Responses are shown separately for rewarded (left) and non-rewarded (right) trials for each Unit. (b) Average normalized firing rates for each Unit Type, across rewarded and non-rewarded trials. The number of units for each type can be found in Table I. Horizontal bars at the bottom of each panel indicate temporal periods during which rewarded trials differed from non-rewarded (generalized linear mixed models, $p<0.05$ ).

Table I Unit Types by Treatment Group

\begin{tabular}{lccc}
\hline & Controls & EtOH-low & EtOH-high \\
\hline Total & 159 & 167 & 135 \\
Type 1 & $60(38 \%)$ & $41(25 \%)^{* * *}$ & $43(32 \%)^{* * *}$ \\
Type 2 & $45(28 \%)$ & $60(36 \%)^{* \# \#}$ & $22(16 \%)^{* * *}$ \\
Type 3 & $9(6 \%)$ & $16(10 \%)$ & $15(11 \%)$ \\
Type 4 & $26(16 \%)$ & $37(22 \%)$ & $27(20 \%)$ \\
Type 5 & $19(12 \%)$ & $13(8 \%)^{\# \#}$ & $28(21 \%)$ \\
\hline
\end{tabular}

Asterisks (*) indicate difference from Controls, while hashes (\#) indicate difference from $\mathrm{EtOH}$-high. The number of symbols indicates the level of significance (one: $p \leqslant 0.05$, two: $p \leqslant 0.01$ ).

of -0.03 for anterior, CI: -0.10 to 0.03$)$. In addition, risk preference was correlated with the magnitude of neural response during this epoch on Win trials (increase in preference for risky lever of $1.7 \%$ for every 0.1 increase in firing rate, $\mathrm{CI}: 0.6-2.7 \%, \quad(\mathrm{~F}(1,141)=5.55, \quad p<0.05))$.
However, these relationships depended on consumption group. The positive relationship between neural response and reward outcome was reduced with higher $\mathrm{EtOH}$ consumption (change in neural response of -0.20 for EtOH-low and -0.30 for EtOH-high, CI: -0.12 to -0.28 and -0.18 to -0.42 , respectively, $(\mathrm{F}(1,428)=23.65$, $p<0.0001)$ ). Consumption group was also related to a larger increase in risk preference, in addition to that accounted for by neural response (increase of $15.0 \%$ for EtOH-low and $22.5 \%$ for EtOH-high, CI: $3.5-26.6 \%$ and $5.2-39.9 \%$, $(\mathrm{F}(1,141)=6.60, p=0.01))$.

\section{DISCUSSION}

The data shown here demonstrate that adolescent ethanol intake results in increased preference for large, risky over small, certain rewards during adulthood, concurrent with altered patterns of activity in the orbitofrontal cortex. While several patterns of OFC responses during performance of the risk task were identified, only one type of neurons showed 

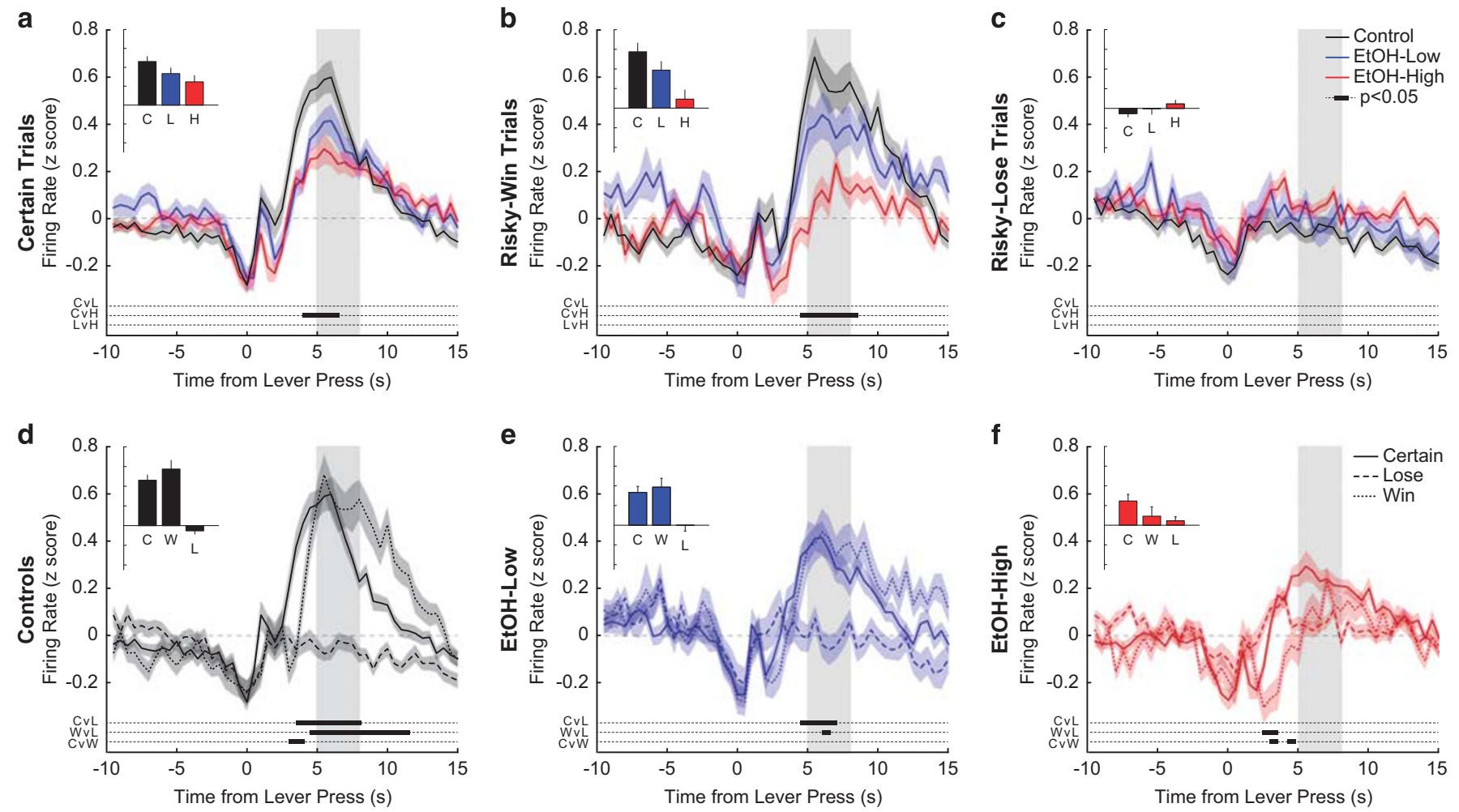

Figure 4 Patterns of normalized firing rates of Type I units, grouped by outcome (a-c) or consumption group ( $d-f$ ). All data are aligned to the time of lever press/choice (time $=0$ ). Reward delivery (or omission) immediately followed the lever press. Horizontal bars at the bottom of each panel indicate temporal periods in which response rates differed between treatment groups (generalized linear mixed models, $p \leqslant 0.05$; panels $\mathrm{a}-\mathrm{C}$ : $\mathrm{CvL}=\mathrm{Control} v \mathrm{vs} \mathrm{EtOH}$-low; $\mathrm{CvH}=$ Control vs EtOH-high; $\mathrm{LvH}=\mathrm{EtOH}$-low vs EtOH-high; panels $\mathrm{d}-\mathrm{f}$ : CVL=Certain vs Lose; WvL = Win vs Lose; CvW = Certain vs Win). Inset figures represent the average firing rates from +5 to $+8 \mathrm{~s}$ following lever press and reward delivery/omission ( $y$ axis scale matches the associated primary figure). Averages are calculated from all 60 Type I neurons from Controls, 4 I from EtOH-low, and 43 from EtOH-high animals. (a) Certain choice trials. Following reward delivery, EtOH-high animals showed reduced activity levels compared with Control animals ( +4 to +6.5 s; all corrected $p \leqslant 0.03)$. (b) Risky choice trials in which large reward was delivered (Win). EtOH-high animals again showed significantly reduced firing rates during the post-reward period compared with Control (+4.5 to +10 s; all corrected $p \leqslant 0.03)$. (c) Risky choice trials in which reward was omitted (Lose). No group differences in firing rates were evident in this condition. (d) Control animals across outcomes. Control animals show elevated levels of activity for both Certain and Win outcomes during the post-press period (Certain: +3.5 to +8.5 s; Wins: +4.5 to II $.5 \mathrm{~s}$; all corrected $p \leqslant 0.0 \mathrm{I}$ ), compared with Lose outcomes. In addition, higher firing rates were seen following Certain outcomes than Wins during a short period following lever press ( +3 to $+4 \mathrm{~s}$; all corrected $p \leqslant 0.0 \mathrm{l})$. (e) EtOH-low animals across outcomes. EtOHlow animals showed patterns of activity similar to Control animals, but slightly blunted in magnitude. Again, elevated firing rates were seen following Certain and Win outcomes compared to Lose (Certain: +4.5 to $+7 \mathrm{~s}$; Wins: +6 to $+6.5 \mathrm{~s}$; all corrected $p \leqslant 0.05$ ), but firing rates did not differ between Certain and Win outcomes. (f) EtOH-high animals across outcomes. EtOH-high animals showed disrupted patterns of activity, with no statistical difference in the encoding of Certain and Lose outcomes. Wins were encoded with decreased activity compared to both Certain and Lose outcomes (Certain: +3 to $+3.5 \mathrm{~s}$ and +4.5 to $+5 \mathrm{~s}$; Lose: +2.5 to $+3.5 \mathrm{~s}$; all corrected $p \leqslant 0.03$ ).

differences related to reward outcome and ethanol treatment. We hypothesized that different patterns of OFC activity, with greatest modulations for Risky Wins, would be associated with higher risk preference in our task, as previously observed by our lab (Roitman and Roitman, 2010). However, our results suggested that the level of voluntary ethanol consumption during adolescence modulated the relationships between reward outcome and OFC activity and between OFC activity and risk preference. Higher levels of consumption were indeed associated with increased risk preference. However, higher risk preference was associated with reduced responses to rewards in one identified population of neurons. The Control animals showed similar risk preference and similar patterns of OFC activity to those we have observed previously in 'risk-neutral' animals (Roitman and Roitman, 2010). However, four of the five task-responsive populations of neurons reported here were not differentially affected by ethanol intake. This departure from our previous finding of elevated OFC activity associated with higher risk preference is confounded by a number of factors, including differences in task design and analysis methods, and perhaps most importantly, prior alcohol consumption. Regardless, it is possible that there are multiple paths to risk preference that may result from heightened reward sensitivity or reduced sensitivity to loss.

The strength of our findings is somewhat surprising, considering the moderate amount of alcohol consumed by our EtOH-high animals. The BELs that resulted from our exposure model are at the threshold for what can be considered 'binge drinking' (Courtney and Polich, 2009). Many models of adult and adolescent alcohol abuse utilize significantly higher levels of ethanol consumption or administration, but our results suggest that such high levels of exposure may not be necessary for persistent effects on 
higher level cognitive abilities, such as risk preference. While our gelatin-based model of alcohol exposure may not result in comparatively high levels of ethanol intake, it does provide stable daily consumption and may model more continuous, low level intake. It is unclear if higher levels of exposure, whether self-administered or experimenter-administered, would have the same effect as those reported here, but additional studies using alcohol preferring stains of rats or alternative access paradigms should be considered. Still, it is alarming that even moderate levels of intake, when consumed chronically during adolescence, exerted significant effects on both behavior and neurophysiology.

While the gelatin vehicle used here is becoming increasingly common (Clark et al, 2012; Nasrallah et al, 2009, 2011; Peris et al, 2006), it is still a relatively new consumption model. In our hands, animals consuming control gelatin ate considerably more gelatin than both the alcohol groups. Differential consumption among groups is unfortunately a common byproduct of many alcohol self-administration models, and considering the caloric content of the gelatin mixture, could result in differential effects on reward circuitry. The consumption of high fat or other 'rich' diets can alter reward circuitry (eg, Volkow et al, 2013); however, this requires extended access to these foods (Johnson and Kenny, 2010), resulting in differential weight gain between groups. We did not observe any differences in body weight between groups (during adolescence or in adulthood), which therefore suggests that even the 'high' levels of gelatin consumption by our control animals were not high enough to result in physiological differences between groups. Regardless, this topic warrants further investigation.

In our task, the probability of risky reward was varied randomly from session-to-session, but held constant within a session. This is an important departure from prior iterations of this task (Cocker et al, 2012; Nasrallah et al, 2009), which have relied on a step-wise reduction in the probability of risky reward across sessions. Our version of the task reduces the potential role of impaired behavioral flexibility on task performance. Despite the random order of sessions, our animals clearly demonstrated effective behavioral adaptation to the changing contingencies. All animals, regardless of group, showed the expected reduction in risk preference when the probability of risky reward becomes less ideal. In the lowest probability condition (12.5\%), all animals rarely, if ever, chose the risky option, indicating that even EtOH-high were capable of avoiding the risky lever when a loss was highly likely.

We found little evidence for a predictive role of OFC during the choice period of the task. Although many unit types changed their firing rate during the pre-press period, no neuron type identified here showed a clear difference in activity during the pre-lever-press period dependent on what choice the animal finally made. Instead, patterns of activity shown here demonstrate a role for the OFC in encoding the outcome of the choice (post-press period). Considering the reward-selective nature of its firing (see Figure 3b), this might suggest that reward is playing some role in its modulation. However, reward encoding in the OFC differs dramatically from reward encoding in regions more typically associated with reward, such as the Nucleus Accumbens (NAc), both in timing relative to reward delivery and encoding of reward size. These differences in the patterns of activation between OFC and NAc neurons suggest that they encode different elements of the reward process, thus differentially contributing to behavioral performance.

Of the five unit types identified here, only one population of neurons showed different activity levels among the three treatment groups. Electrophysiological recordings did not allow for the biomolecular identification of the neurons belonging to this Unit Type, and should be the focus of future studies. Considering adolescent alcohol's impact on reward encoding in the NAc in a similar task (Nasrallah et al, 2011), it is likely that the alterations we report here may be functionally related to mis-codings of reward downstream of the OFC. The OFC is known to project to reward-encoding regions, such as the NAc and Ventral Tegmental Area. Using a similar behavioral model to the one used here, dopamine release in the NAc in response to risky rewards has been shown to be increased in ethanol consuming animals (Nasrallah et al, 2011). Therefore, the altered OFC neuronal population shown here may be inhibitory interneurons. The reduced level of neuronal activity in EtOH-high animal may be releasing inhibition on the excitatory projections from the OFC to reward circuitry, enhancing dopamine signaling. Further investigation of the identity and role of this population in risk preference, and how it is affected by exposure to alcohol during adolescence, is clearly warranted.

One issue left unclear by the current set of experiments, and the field as a whole, is whether ethanol consumption in adolescence alters neurobiology, thus changing cognitive performance, or if innately different neurobiologies, and therefore innately different cognitive abilities, promote greater adolescent ethanol consumption. Such data are of immense importance to understanding the actual impact of alcohol intake during this critical period. If alcohol consumption is driven by an innate predisposition for risky outcomes, then our Control group would have consisted of both risk takers and risk avoiders, and we might have expected the behavior of Controls to be approximated by the average behavior and OFC activity of the EtOH-high and EtOH-low animals; however, this was not the case. In addition, recent work with Long-Evans rats has found that pre-existing impulsivity does not predict later alcohol selfadministration (Stein et al, 2015). Regardless of whether such pre-existing differences in neurobiology existed, the blunting of OFC responses occurred only in animals with higher levels of consumption. Thus, the differences observed here may suggest that even with potential pre-existing biases, alcohol consumption can exacerbate behavioral biases via a reduction in OFC sensitivity to reward.

One additional issue left unclear is the role of early life stress on animals' alcohol consumption and risk preference. All animals were handled identically, exposed to gelatin for the same period of time, and behaviorally tested at the same time points. However, it is likely that the differential patterns of alcohol consumed by our EtOH-high and EtOH-low animals are indicative of underlying neurophysiological differences, perhaps resulting from differential early life stress. Alternatively, it is also possible that adolescent alcohol consumption exacerbates the impact of early life stress. Such stress could be the result of a number of uncontrolled aspects of the study, including shipment from the breeding facility (Charles River) to the laboratory and differences in maternal care at the breeding facility. Early life stress clearly has an 
important role in both impulsivity (Lovallo, 2013; Lovallo et al, 2013) and drug effects (Enoch, 2011), and thus warrants additional study to determine what aspects of the results presented here may be attributable to early life stress, alcohol, or the interaction between the two.

The data we present here demonstrate a relationship between adolescent alcohol intake, increased risk preference, and altered function of the orbitofrontal cortex. Importantly, we identify a highly specific association between alcohol intake and the function of this brain region, restricted to one OFC response pattern and the encoding of one element of the behavioral task. This highly specific relationship persisted across an extended period during which no alcohol exposure occurred. As such, this may be reflective of an altered developmental trajectory of OFC, likely due to the alcohol exposure itself. Despite its restrictive nature, we cannot underestimate the importance of the function of this region, since it is involved in the encoding of rewards, and therefore involved in goal-directed decision-making. Thus, adolescent alcohol consumption may drive alterations in additional cognitive abilities through such increases in hypofrontality, perhaps also increasing risk for depression and other psychiatric disorders.

\section{FUNDING AND DISCLOSURE}

Funding for this project was provided for by a Fay Frank Seed Grant from the Brain Research Foundation (JDR). The authors declare no conflict of interests.

\section{ACKNOWLEDGMENTS}

We would like to thank Christopher Sinon and Alyssa Secreto for their assistance with data collection, and Dr Darin Knapp for his assistance with blood ethanol level quantification.

\section{REFERENCES}

Bava S, Tapert SF (2010). Adolescent brain development and the risk for alcohol and other drug problems. Neuropsychol Rev 20: 398-413.

Bell RL, Rodd ZA, Sable HJK, Schultz JA, Hsu CC, Lumeng L et al (2006). Daily patterns of ethanol drinking in peri-adolescent and adult alcohol-preferring (P) rats. Pharmacol Biochem Behav 83: 35-46.

Bell RL, Rodd ZA, Smith RJ, Toalston JE, Franklin KM, McBride WJ (2011). Modeling binge-like ethanol drinking by peri-adolescent and adult $\mathrm{P}$ rats. Pharmacol Biochem Behav 100: 90-97.

Clark JJ, Nasrallah NA, Hart AS, Collins AL, Bernstein IL, Phillips PEM (2012). Altered risk-based decision making following adolescent alcohol use results from an imbalance in reinforcement learning in rats. PLoS One 7: e37357.

Cocker PJ, Dinelle K, Kornelson R, Sossi V, Winstanley CA (2012). Irrational choice under uncertainty correlates with lower striatal $\mathrm{D}(2 / 3)$ receptor binding in rats. $J$ Neurosci 32: 15450-15457.

Courtney K, Polich J (2009). Binge drinking in young adults: data, definitions, and determinants. Psychol Bull 135: 142-156.
Crews FT, He J, Hodge C (2007). Adolescent cortical development: a critical period of vulnerability for addiction. Pharmacol Biochem Behav 86: 189-199.

Crews FT, Mdzinarishvili A, Kim D, He J, Nixon K (2006). Neurogenesis in adolescent brain is potently inhibited by ethanol. Neuroscience 137: 437-445.

Doremus-Fitzwater TL, Varlinskaya EI, Spear LP (2010). Motivational systems in adolescence: possible implications for age differences in substance abuse and other risk-taking behaviors. Brain Cogn 72: 114-123.

Enoch M-A (2011). The role of early life stress as a predictor for alcohol and drug dependence. Psychopharmacology 214: $17-31$.

Hsu M, Bhatt M, Adolphs R, Tranel D, Camerer CF (2005). Neural systems responding to degrees of uncertainty in human decisionmaking. Science 310: 1680-1683.

Johnson PM, Kenny PJ (2010). Dopamine D2 receptors in addiction-like reward dysfunction and compulsive eating in obese rats. Nat Neurosci 13: 635-641.

Kepecs A, Uchida N, Zariwala HA, Mainen ZF (2008). Neural correlates, computation and behavioural impact of decision confidence. Nature 455: 227-231.

Lovallo WR (2013). Early life adversity reduces stress reactivity and enhances impulsive behavior: implications for health behaviors. Int J Psychophysiol 90: 8-16.

Lovallo WR, Farag $\mathrm{NH}$, Sorocco $\mathrm{KH}$, Acheson A, Cohoon AJ, Vincent AS (2013). Early life adversity contributes to impaired cognition and impulsive behavior: studies from the Oklahoma Family Health Patterns Project. Alcohol Clin Exp Res 37: 616-623.

McDannald MA, Esber GR, Wegener MA, Wied HM, Liu T-L, Stalnaker TA et al (2014). Orbitofrontal neurons acquire responses to "valueless" Pavlovian cues during unblocking. eLife 3: $\mathrm{e} 02653$.

McMurray MS, Amodeo LR, Roitman JD (2014). Effects of voluntary alcohol intake on risk preference and behavioral flexibility during rat adolescence. PLoS One 9: e100697.

Nasrallah NA, Clark JJ, Collins AL, Akers CA, Phillips PEM, Bernstein IL (2011). Risk preference following adolescent alcohol use is associated with corrupted encoding of costs but not rewards by mesolimbic dopamine. Proc Natl Acad Sci USA 108: 5466-5471.

Nasrallah NA, Yang TWH, Bernstein IL (2009). Long-term risk preference and suboptimal decision making following adolescent alcohol use. Proc Natl Acad Sci USA 106: 17600-17604.

National Institute on Alcohol Abuse and Alcoholism (2004). NIAAA council approves definition of binge drinking. NIAAA Newsl 3: 3.

Nixon K, Morris SA, Liput DJ, Kelso ML (2010). Roles of neural stem cells and adult neurogenesis in adolescent alcohol use disorders. Alcohol 44: 39-56.

Padoa-Schioppa C, Cai X (2011). The orbitofrontal cortex and the computation of subjective value: consolidated concepts and new perspectives. Ann N Y Acad Sci 1239: 130-137.

Peris J, Zharikova A, Li Z, Lingis M, MacNeill M, Wu MT et al (2006). Brain ethanol levels in rats after voluntary ethanol consumption using a sweetened gelatin vehicle. Pharmacol Biochem Behav 85: 562-568.

Roitman JD, Roitman MF (2010). Risk-preference differentiates orbitofrontal cortex responses to freely chosen reward outcomes. Eur J Neurosci 31: 1492-1500.

Selemon LD (2013). A role for synaptic plasticity in the adolescent development of executive function. Transl Psychiatry 3: e238. 
Stalnaker TA, Cooch NK, McDannald MA, Liu T-L, Wied H, Schoenbaum G (2014). Orbitofrontal neurons infer the value and identity of predicted outcomes. Nat Commun 5: 3926.

Stein JS, Renda CR, Barker SM, Liston KJ, Shahan TA, Madden GJ (2015). Impulsive choice predicts anxiety-like behavior, but not alcohol or sucrose consumption, in male Long-Evans rats. Alcohol Clin Exp Res 39: 932-940.
Tobler PN, Christopoulos GI, O’Doherty JP, Dolan RJ, Schultz W (2009). Risk-dependent reward value signal in human prefrontal cortex. Proc Natl Acad Sci USA 106: 7185-7190.

Volkow ND, Wang G-J, Tomasi D, Baler RD (2013). The addictive dimensionality of obesity. Biol Psychiatry 73: 811-818.

Supplementary Information accompanies the paper on the Neuropsychopharmacology website (http://www.nature.com/npp) 